\title{
Los efectos de la pandemia de COVID-19 en enclaves arqueológicos al aire libre en Galicia
}

\author{
Estrela C. García García | Graduada en Bellas Artes \\ URL de la contribución <www.iaph.es/revistaph/index.php/revistaph/article/view/4835>
}

\section{RESUMEN}

Tras el primer impacto de la pandemia de la enfermedad COVID-19, se analiza el efecto que esta tuvo en el periodo estival del año 2020 en lugares o centros culturales con arte rupestre o yacimientos arqueológicos de la prehistoria reciente al aire libre y cerrados, ubicados en la Comunidad Autónoma de Galicia.

Acercándose a la premisa de que el modelo de visita del turismo arqueológico al aire libre puede ser una vía de futuro para remodelar el modelo turístico previo a la pandemia, basado en los flujos masivos de personas, frente a un modelo más sostenible, equilibrado y adaptado a las nuevas medidas sociosanitarias, se revisa el flujo de visitantes entre los meses de junio y septiembre para examinar cuál ha sido el comportamiento del público en este nuevo contexto tras la primera ola de la pandemia, el primer confinamiento domiciliario y el proceso de desescalada.

Los resultados se analizan teniendo en cuenta estos datos y otros factores que han sido claves en ese contexto como son la presencia en redes de las instituciones culturales y las restricciones de movilidad, entre otros. Se ha podido comprobar que, efectivamente, los centros al aire libre tuvieron un menor descenso de visitas, lo cual demuestra que su modelo turístico y las características intrínsecas que los definen han sido clave para atraer al visitante en un contexto de emergencia sanitaria mundial; lo que también puede ser un fuerte indicador de un cambio de rumbo en la dirección en la cual el arqueoturismo se produce, abandonando el modelo de turismo de masas a uno más sostenible y pausado.

\section{Palabras clave}

Arqueoturismo | COVID-19 | Galicia | Museos | Patrimonio arqueológico | Patrimonio cultural | Turismo cultural | Visitantes | 


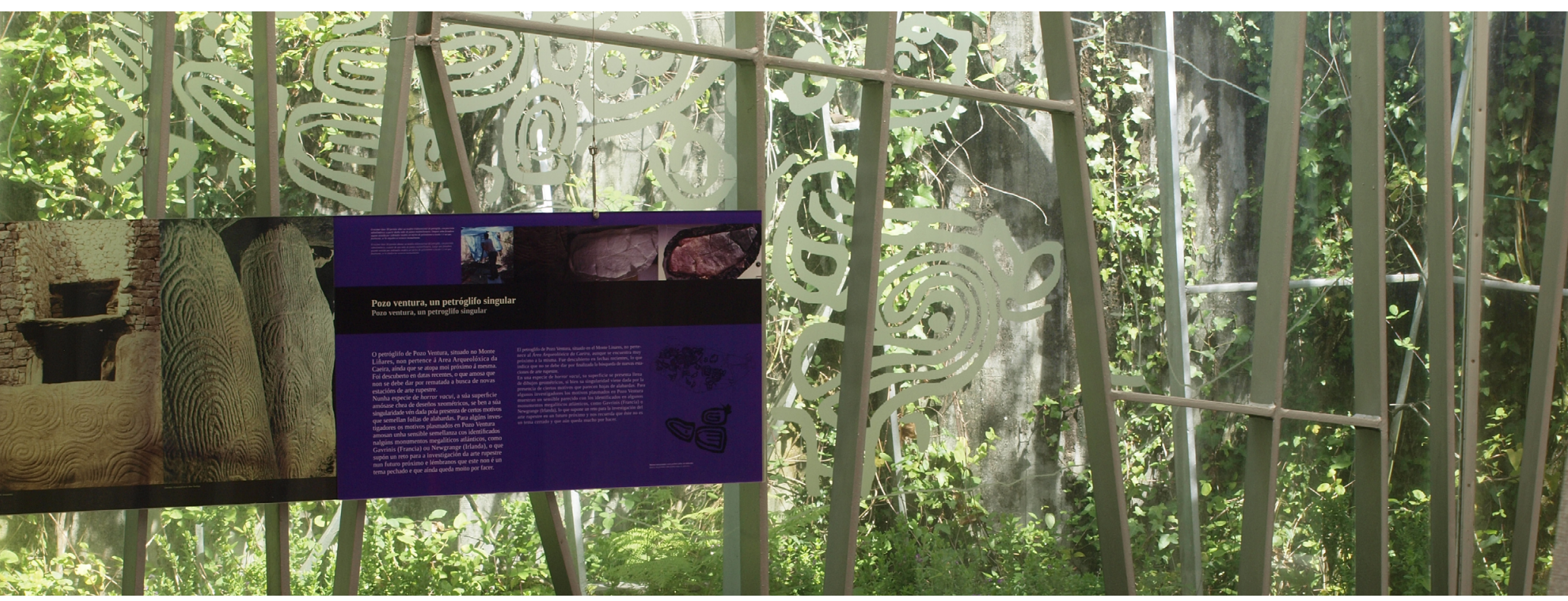

\section{The effects of the COVID-19 pandemic on open air archaeological sites in Galicia}

\section{ABSTRACT}

After the first impact of the COVID-19 disease pandemic, we analyse the impact it had in the summer period of 2020 on sites or cultural centres with rock art or archaeological sites of recent prehistory in the open air and closed, located in the Autonomous Community of Galicia. In view of the premise that the open-air archaeological tourism visit model could be a future way of remodelling the tourism model prior to the pandemic, based on massive flows of people, as opposed to a more sustainable, balanced model adapted to new social and health measures, the flow of visitors between the months of June and September is reviewed to analyse the behaviour of the public in this new context after the first wave of the pandemic, the first home lockdown and the process of de-escalation. The results are analysed taking into account these data and other factors that have been key in this context, such as the presence of cultural institutions in networks and mobility restrictions, among others. It has been possible to verify that, indeed, the open-air centres had a lower drop in visits, which shows that their tourism model, and the intrinsic characteristics that define them, have been key to attracting visitors in a context of global health emergency; which may also be a strong indicator of a change of direction in the direction in which archaeotourism takes place, abandoning the model of mass tourism to a more sustainable and less intensive one.

\section{Key words}

Archeotourism | COVID-19 | Galicia (Spain) | Museums | Archaeological heritage | Cultural heritage | Cultural tourism | Visitors |

Cómo citar: García García, E. (2021) Los efectos de la pandemia de COVID-19 en enclaves arqueológicos al aire libre en Galicia. Revista PH, n. ${ }^{\circ}$ 103, pp. 80-104. Disponible en: www.iaph.es/revistaph/index.php/revistaph/article/view/4835 DOI 10.33349/2021.103.4835

Enviado: 19/01/2021 | Aceptado: 13/05/2021 | Publicado: 10/06/2021 


\section{INTRODUCCIÓN}

La pandemia del SARS-COV-2, que ha afectado al mundo de forma nunca antes vista, ha puesto a prueba la capacidad del sector turístico y patrimonial para responder ante una situación de crisis sin precedentes.

La enorme labor de adaptación y respuesta rápida de la cultura a nivel internacional ha sido admirable, ya que en la mayor parte de estos espacios el cierre obligatorio debido a los confinamientos supuso una situación de enorme incertidumbre.

Se estima que un $54 \%$ de los países han cerrado monumentos y sitios Patrimonio de la Humanidad en marzo de 2020 (Unesco 2020c, 1), lo que ha conllevado una coyuntura única en la historia de la gestión del patrimonio, en la cual el futuro incierto dejaba al sector de la cultura ante un porvenir dudoso, sin protocolos establecidos o modelos de actuación que pudieran servir de guía ante una crisis sanitaria de tales características.

Con el fin del confinamiento domiciliario de la primera ola, el objetivo principal de los museos y centros culturales fue lograr un equilibrio entre mantener las medidas sanitarias para garantizar la seguridad tanto de visitantes como de personal trabajador, y poder permanecer abiertos siempre que las circunstancias lo permitieran.

Y aunque las medidas sanitarias se deben seguir en cualquier sitio, sí que se aprecian diferencias en distintos lugares en los cuales se pueden visitar bienes culturales y arqueológicos, y en este aspecto los lugares abiertos o al aire libre tienen una ventaja indiscutible frente a lugares cerrados, que recae principalmente en la capacidad de ventilación y en la facilidad de mantenimiento de las distancias interpersonales.

En concreto, nos centraremos en la huella que la pandemia ha dejado en lugares con arte rupestre o yacimientos arqueológicos de la prehistoria en Galicia, tanto cerrados como al aire libre y, a través del número de visitantes de público general, veremos cómo han afectado estas circunstancias a las visitas durante el periodo de verano del año 2020.

El patrimonio arqueológico en Galicia es uno de los bienes culturales que más valor tienen en la comunidad, en concreto son especialmente conocidas las fortificaciones de la Edad del Hierro de la cultura castreña, mejor conocidos como castros, así como los grabados rupestres prehistóricos, o petroglifos, que se esparcen por todo el territorio gallego, alcanzando fronteras de otras comunidades como León o países vecinos como Portugal, y que guardan relación histórica y formal con muchas otras localizaciones de la Europa atlántica. 
El artículo estudiará, a través de la comparación del número de visitantes del periodo estival de 2019 y 2020 de lugares con este tipo de yacimientos prehistóricos en Galicia, cuál ha sido el efecto que la pandemia tuvo en el flujo de visitas y en los centros, y cómo estos últimos se han adaptado a las circunstancias excepcionales vividas.

Cabe mencionar antes de abordar esta cuestión que el número de visitantes no es un indicativo ni de la calidad de un centro ni de la satisfacción o la impresión que este deja en un público, y que como se podrá comprobar, estos números están influenciados por muchos otros factores además de las restricciones o el miedo del público general a acudir a lugares culturales. Se debe también tener en cuenta la escala en la cual trabajan todos los centros que se verán a continuación, ya que no todos cuentan con la misma capacidad de visita o los mismos recursos.

Se indicarán las incidencias particulares que afectaron a estos centros para tomarlas en consideración a la hora de examinar los resultados obtenidos de la comparativa, así como se proporcionará un contexto adecuado para comprender mejor otros factores que influyeron y cambiaron esta situación, como son las restricciones de movilidad o las medidas sociosanitarias establecidas.

\section{EL ARQUEOTURISMO EN ESPAÑA Y EN GALICIA: UNA PEQUEÑA APROXIMACIÓN}

Es fundamental también destacar el concepto de arqueoturismo, que en este caso es el que afecta ya que es la tipología que incumbe al modelo de visita de los yacimientos de la muestra.

El arqueoturismo se puede definir como una modalidad de turismo o visita que está motivada por el interés de conocer el potencial o el valor arqueológico de un yacimiento, a partir del cual se genera una estructura dedicada a la visita del mismo (López Ortega y Collado Moreno 2018, 599).

Se trata de un fenómeno que no es nuevo, y que está ligado a la historia del turismo a lo largo de diferentes épocas, y que, por su naturaleza, tiene una carga cultural mayor, al nacer de un interés del visitante por la cultura y la historia, así como por la arqueología (López Ortega y Collado Moreno 2018).

En el caso español, la mayor parte de yacimientos arqueológicos visitables se localiza en el entorno rural, lo que provoca que este turismo sea un complemento o vaya acompañado de otras visitas a lugares adyacentes, es decir, realizar una excursión al entorno cercano al yacimiento como poblaciones, playas o visitas a espacios naturales, que atraen al turista a estos 
Mapa de la distribución del arqueoturismo en España. 2017 | fuente Fernández Ortea (2017)

Mapa de la distribución del arqueoturismo en Galicia. 2017 | fuente Fernández Ortea (2017)
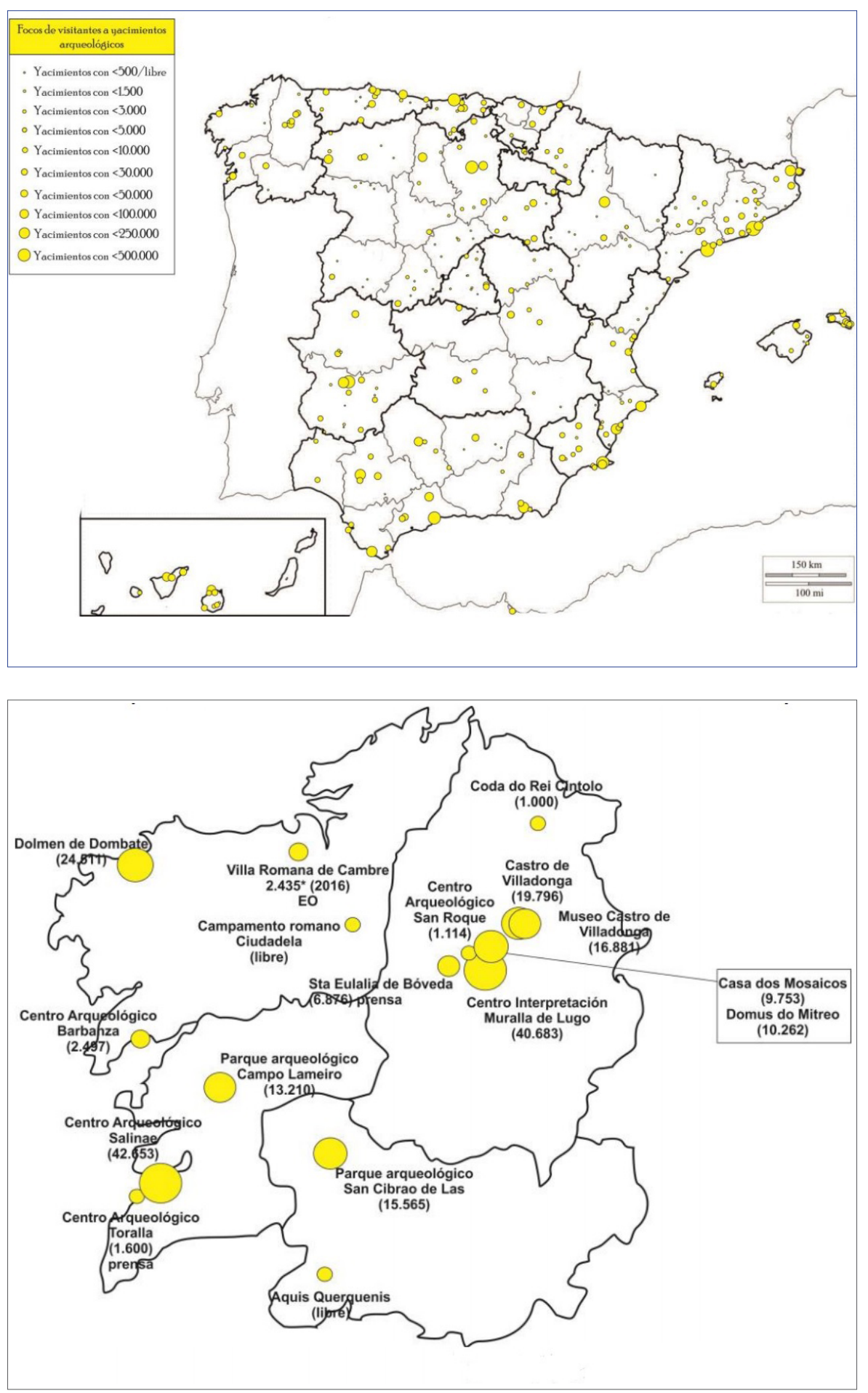
monumentos y generan un impacto notable en el tejido socioeconómico de la zona en la cual se enmarcan (Fernández Ortea 2017).

Estos yacimientos se reparten de forma similar, sin haber grandes concentraciones ni vacíos, por todo el territorio del Estado Español, como se puede apreciar en el mapa de la página anterior, con una concentración algo mayor en el litoral mediterráneo y el cantábrico.

Pasando al caso gallego, podemos comprobar (ver mapa de la p. 84) cómo se reparten estos lugares de manera más o menos equitativa por todo el territorio, aunque se concentran más en la costa atlántica y en Lugo. Esto se debe a la presencia de la muralla romana de Lugo, y los museos y centros de interpretación de la cultura romana repartidos por la ciudad. No obstante, se puede apreciar que, como en el resto de la comunidad, la tipología de yacimiento arqueológico que más destaca junto a la romana es la de monumentos megalíticos y castros, patrimonio arqueológico que ocupa este trabajo.

El impacto de este tipo de turismo en la comunidad gallega representa una pequeña parte en comparación con otras comunidades autónomas como Extremadura o Murcia, suponiendo un 2,74 \% en el año 2014, frente al 12 o $14 \%$ de estas últimas (Fernández Ortea 2017, 92). Hay que señalar una vez más que la tipología de yacimiento que mayor impacto causa, o lo que es lo mismo, que más visitantes atrae a nivel general de todo el país, es el relacionado con monumentos de época romana, lo que deja a la prehistoria en un nivel inferior de impacto en el ámbito nacional. Y paradójicamente, a pesar de encontrarse en mayor medida en entornos rurales, tiene un mayor flujo de visitas en entornos urbanos, debido a su mayor accesibilidad y a ser uno de los grandes puntos de concentración del turismo (Fernández Ortea, 2017).

El arqueoturismo, pues, proporciona una abundante oferta en nuestro país (Fernández Ortea 2017, 102), y supone un recurso muy valioso, pero a la vez poco valorado en gran medida, lo que conlleva una gran pérdida económica y también cultural.

Es importante señalar que estos datos corresponden a un contexto anterior a la pandemia. En el momento presente los números seguramente han fluctuado. A la espera de un estudio detallado centrado en el sector del turismo arqueológico, el miedo al contagio y la búsqueda de espacios diáfanos y al aire libre constituirán factores que jueguen a favor del arqueoturismo, y que muy posiblemente sean los causantes de un aumento en las visitas a este tipo de lugares, lo que favorecerá que cambien las variables que hasta ahora han definido la elección de un destino turístico relacionado con el patrimonio arqueológico. 


\section{EL IMPACTO DE LA PANDEMIA DE COVID-19 EN EL SECTOR CULTURAL EN ESPAÑA}

La presencia de la pandemia se ha dejado notar en todos los sectores de la sociedad a lo largo del globo, siendo el sector cultural uno de los más afectados por las cuarentenas y por las medidas restrictivas sanitarias para paliar el número de contagios, así como por la reticencia del público a acudir a espacios públicos y normalmente aglomerados por temor al contagio.

Es fundamental indicar que, como es lógico, estas condiciones han supuesto un factor clave a la hora de decidir el turista acudir y visitar un bien cultural durante el periodo estival, acrecentado por la movilidad limitada de algunas zonas o incluso la capacidad económica, que sin duda se ha visto mermada durante este año.

Los efectos de estas circunstancias se reflejan en el acusado descenso del número de visitantes. Pese a todo, el sector de la cultura ha sido uno de los más seguros, donde se ha producido el menor número de contagios y brotes desde el inicio de la desescalada y la "nueva normalidad" hasta ahora.

Según los informes semanales del Ministerio de Sanidad y el Centro de Coordinación Nacional de Alertas y Emergencias Sanitarias sobre la evolución y situación de la pandemia en España, a 23 de octubre de 2020, en el sector cultural solamente se habían detectado un total de 5 brotes desde el mes de mayo, tal y como se puede comprobar en la tabla de la página siguiente.

Esto supone un $0,025 \%$ del total de brotes con casos registrados (Blázquez 2020), lo cual indica que, siguiendo las recomendaciones estipuladas y las medidas de protección sanitaria, el sector de la cultura garantiza un grado muy alto de seguridad tanto para el visitante como para el personal trabajador.

A pesar de estos datos tan positivos, este grupo ha sido uno de los más castigados por esta crisis sanitaria y económica, que a nivel mundial representaba antes un $2,6 \%$ del PIB mundial (Unesco 2020c, 2). Tras el cierre generalizado se estima que un $10 \%$ de los museos no han vuelto a abrir sus puertas, problema que se acrecienta en el sector privado, con casi un tercio de las galerías y comerciantes de arte y patrimonio cultural con pocas perspectivas de recuperación tras la crisis (Unesco 2020c, 1).

Este mismo organismo también advierte de la preocupación ante la conservación de sitios y yacimientos arqueológicos, ya que en muchos casos los ingresos procedentes de las entradas del público son la única fuente de ganancias que tienen para poder mantenerse (Unesco 2020c, 1). Según 


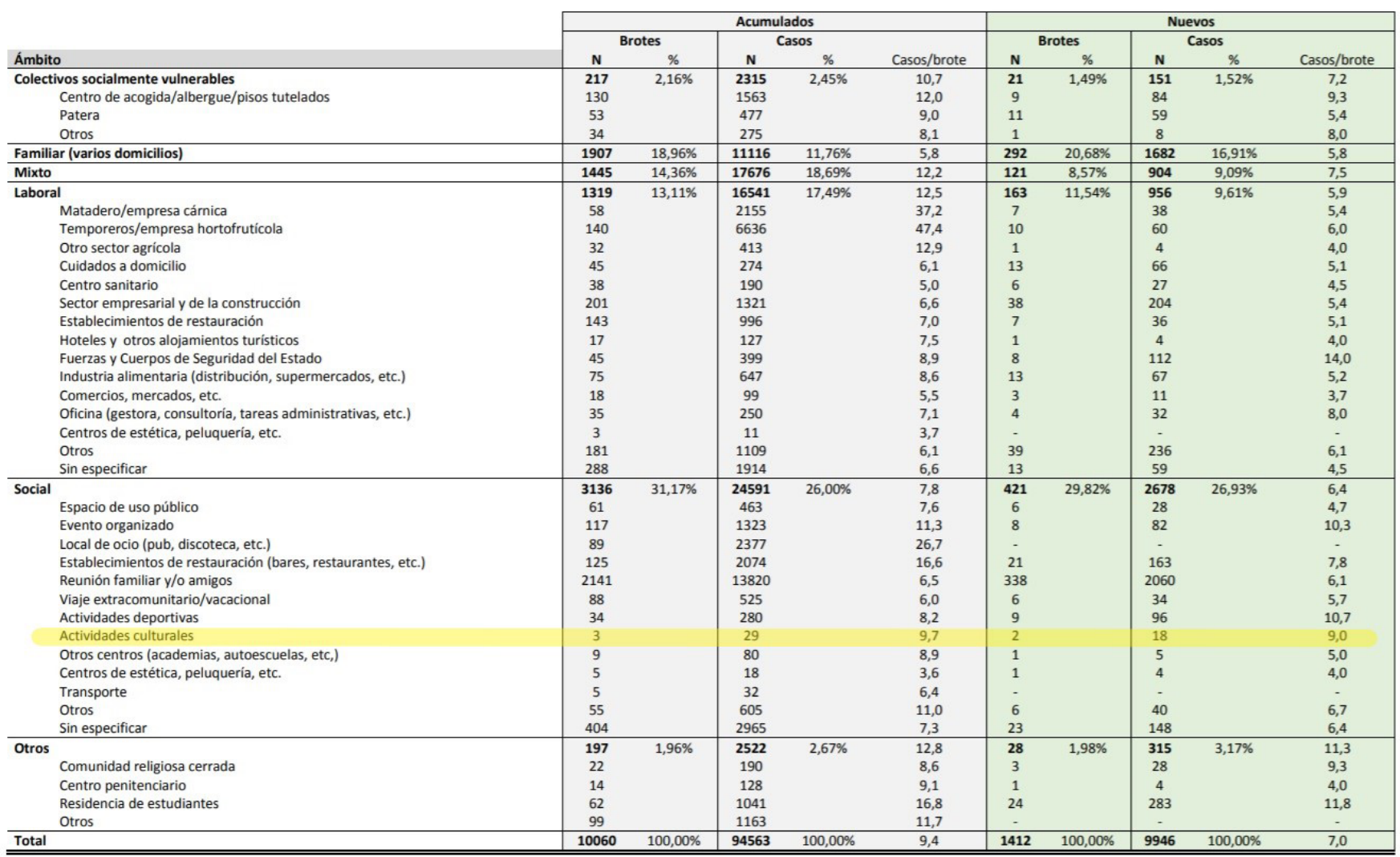

Número de brotes acumulados y nuevos de coronavirus por sectores sociales en los cuales han surgido, resaltando el sector cultural | fuente

la Organización Mundial del Turismo (Unesco 2020a, 2), el turismo cultuMinisterio de Cultura y Deporte, Instituto de Patrimonio Cultural de España (2020) ral representa un $40 \%$ de los ingresos de turismo a nivel global; y la Red de Organizaciones de Museos Europeos indicó en mayo de 2020 que un $75-80 \%$ de los museos que la conforman reportaron pérdidas de ingresos por el cese total del turismo durante el confinamiento domiciliario, y se preveía que continuara durante todo el año 2020, incluyendo la campaña de verano (Unesco 2020a, 2).

En el caso de España, los datos nos muestran que las pérdidas han sido muy grandes tras el confinamiento, lo que dejaba al sector cultural en una posición muy debilitada para afrontar la campaña de verano, que en muchas instancias se ha visto notablemente afectada por las limitaciones y restricciones. Para afrontar estas dificultades, se anunciaron numerosas bolsas y ayudas económicas para tratar de mantener y salvar el sector (Paquete 2020), pero tal y como vemos a continuación en la tabla de la p. 89, no solamente existe una diferencia significativa de una comunidad autónoma a otra en cuanto a su procedencia, lo que genera inseguridad y desinformación, sino que evita una posible asociación interterritorial de centros culturales para actuar en conjunto. 
Pérdidas de ingresos del $2 .^{\circ}$ trimestre de 2020 (abril-junio) en centros y asociaciones culturales en España | fuente Abeledo Sanchís (2020)

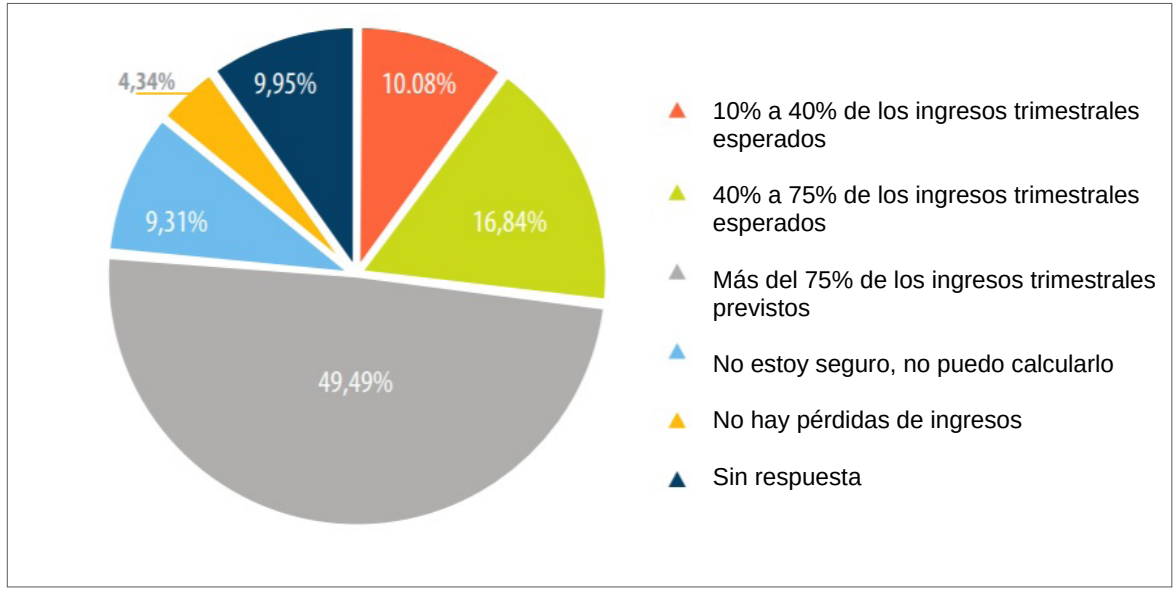

Adicionalmente las organizaciones del sector las consideran insuficientes y abogan por un mayor respaldo público, a lo que se suma el desconocimiento general y la falta de información institucional sobre qué ayudas están disponibles (Abeledo Sanchís 2020).

Como respuesta a esta crisis muchos centros y organizaciones culturales han tenido que tomar medidas para afrontarla; casi la mitad de ellas ha tenido que implementar el teletrabajo, el $42 \%$ reducir su actividad al mínimo en periodo de confinamiento o posterior, y un $12 \%$ ha tenido que ajustar su plantilla debido a las dificultades económicas, cifras que en el caso del patrimonio cultural aumentan a un $62 \%$ de organizaciones que han tenido que reducir su actividad y un $10 \%$ las que ha tenido que prescindir de parte de la plantilla (Abeledo Sanchís 2020).

Desde el sector se reclama más apoyo institucional para recuperar la confianza perdida y que la pandemia ha ocasionado, así como la elaboración de un plan estratégico de gestión y recuperación dedicado exclusivamente al sector creativo y cultural, tal y como se ha hecho con otros sectores (Abeledo Sanchís 2020).

Esta situación propició el aumento del uso de actividades digitales u online, y la promoción de museos y centros culturales que se han mantenido cerrados durante la cuarentena de los meses de marzo, abril y mayo de 2020 a través de las redes sociales, como ya hemos mencionado, incrementando su actividad online para evitar perder el contacto con el público y procurar futuras visitas, además de otras actividades como la revisión del catálogo o limpieza.

Muchos de los emplazamientos seleccionados para este trabajo han tenido una notable presencia en redes durante los meses de la desescalada y la 


\begin{tabular}{|l|c|c|c|c|c|c|}
\cline { 2 - 7 } & \multicolumn{4}{|c|}{} & \multicolumn{2}{c|}{ Públicas } \\
\cline { 2 - 7 } & $\begin{array}{c}\text { Unión } \\
\text { Europea }\end{array}$ & $\begin{array}{c}\text { Gobierno } \\
\text { nacional }\end{array}$ & $\begin{array}{c}\text { Gobierno } \\
\text { autonómico }\end{array}$ & $\begin{array}{c}\text { Gobierno } \\
\text { local }\end{array}$ & Fundaciones & Asociaciones \\
\hline $\begin{array}{l}\text { Comunitat } \\
\text { Valenciana }\end{array}$ & $4 \%$ & $66 \%$ & $58 \%$ & $44 \%$ & $2 \%$ & $10 \%$ \\
\hline $\begin{array}{l}\text { Madrid + } \\
\text { Catalunya }\end{array}$ & $17 \%$ & $70 \%$ & $48 \%$ & $17 \%$ & $13 \%$ & $9 \%$ \\
\hline Canarias & $0 \%$ & $42 \%$ & $46 \%$ & $17 \%$ & $4 \%$ & $8 \%$ \\
\hline Galicia & $0 \%$ & $93 \%$ & $13 \%$ & $0 \%$ & $0 \%$ & $33 \%$ \\
\hline $\begin{array}{l}\text { Resto CCAA } \\
\text { (ESP) }\end{array}$ & $2 \%$ & $80 \%$ & $40 \%$ & $27 \%$ & $2 \%$ & $13 \%$ \\
\hline
\end{tabular}

campaña de verano de 2020 para atraer visitantes y anunciar las actividades adaptadas a las medidas sanitarias y de distanciamiento social, como podemos ver en el ejemplo de la imagen de la derecha, en el cual el Parque Arqueológico del Arte Rupestre (PAAR) anuncia el protocolo que se llevará a cabo para programar las actividades del verano, además de proporcionar recursos de medios de comunicación informando sobre el mismo.

La presencia en redes ha sido fundamental para los centros culturales en muchos casos para poder mantenerse a flote y conectar con el público durante el periodo de confinamiento domiciliario, programando nuevas actividades online para sustituir a los eventos y exposiciones presenciales que se tuvieron que cancelar.

Según datos de la Red de Organizaciones de Museos Europeos, un 40 \% de los museos en Europa "detectaron un incremento en las visitas en línea" (Unesco 2020b, 6), lo que demuestra que la necesidad de consumo cultural en los meses de confinamiento domiciliario fue importantísima, y lo que auguró el retorno del público en cuanto se efectuara la reapertura.

A su vez, la difusión en otros medios como la prensa escrita también ha jugado un papel determinante en este objetivo, alcanzando una audiencia mayor con la meta de aumentar el número de visitas en una campaña de verano que se preveía más baja de lo habitual por la presencia de la pandemia, así como por el constante riesgo de un nuevo confinamiento o de medidas restrictivas más duras de lo esperado.

Esta presencia, acompañada con una fuerte promoción de las actividades programadas en verano, junto con la información de las medidas sanitarias implementadas, ha ayudado a atraer visitantes que, de otra forma, no hubieran acudido a estos centros en el periodo estival.

Porcentaje de ayudas disponibles para el sector cultural en España por comunidades | fuente Abeledo Sanchís (2020)

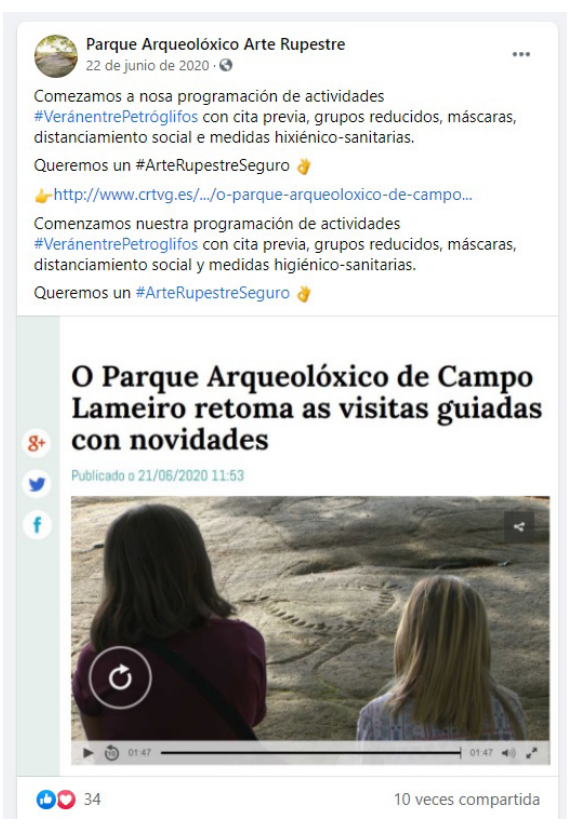

Perfil de Facebook del PAAR en el que se anuncia la programación de las actividades de verano 2020 


\section{LAS RECOMENDACIONES OFICIALES PARA LA REAPERTURA $Y$ GESTIÓN DE LOS BIENES CULTURALES TRAS LA DESESCALADA}

Para enfrentar esta situación de reapertura tras el confinamiento, y como parte del plan de desescalada y llegada a la denominada nueva normalidad, en mayo de 2020 el Ministerio de Cultura y Deporte y el Instituto del Patrimonio Cultural de España -a partir de ahora IPCE- publicaron una guía de actuación y recomendaciones para la gestión del patrimonio cultural ante la crisis sanitaria. En este documento se detallan las medidas a tener en cuenta para la reapertura y gestión segura de los centros culturales siguiendo unas pautas adecuadas a cada tipo de manifestación.

Existen recomendaciones generales y específicas para cada tipo de bien patrimonial. Las generales cuentan con medidas dedicadas a la salud de las plantillas, el público y el buen estado de los bienes, ahora veremos un pequeño resumen de estas (Ministerio de Cultura y Deporte, Instituto del Patrimonio Cultural de España 2020, 7-10):

1. Comprobar el estado de salud de las plantillas a través de pruebas diagnósticas.

2. Garantizar la seguridad de las plantillas con equipos y materiales de protección individual.

3. Medidas de protección adaptando el espacio de trabajo a la continua desinfección y mantenimiento de las distancias interpersonales.

4. Estudio de la capacidad física de los espacios: ajustando el aforo para asegurar la distancia interpersonal y reducirlo en los distintos lugares del centro si fuera necesario, resaltando el papel fundamental de la ventilación y del uso de mascarillas.

5. Desinfección y limpieza en espacios o zonas de contacto compartido.

6. Se debe velar por la seguridad del público visitante, asegurando el uso de la mascarilla, y eliminando cualquier elemento reutilizable que pueda suponer un foco de contagio.

7. Control del contacto con los bienes culturales, limitando el contacto entre el personal trabajador y el público, así como de éstos con los bienes que se exponen, ya que la situación exige nuevas medidas de desinfección que pueden ser abrasivas para los bienes.

8. Se recomienda realizar una revisión de los protocolos de visitas en favor de otros más favorables con la conservación del patrimonio cultural. 
9. Al igual que en el punto anterior, se anima a los centros expositivos a revisar los protocolos de previsión y de emergencias e incluir situaciones como la actual, que se base en las experiencias vividas en este periodo.

Para ello el IPCE proporciona unas plantillas de Excel para evaluar los diferentes bienes culturales y su supervisión (Ministerio de Cultura y Deporte, Instituto del Patrimonio Cultural de España 2020, 10).

La lista es extensa y detallada, e incluye todos los aspectos a tener en cuenta para la correcta actuación de los centros culturales, además de dejar espacio para adaptarse de la manera más adecuada dependiendo de las características de cada lugar concreto.

Ante esta disyuntiva los conjuntos arqueológicos al aire libre han representado una excepción, ya que sus características permiten la realización de actividades y mantener de manera adaptada su programa, visitas guiadas y de presencia de público en el yacimiento.

Estos bienes culturales han sido de los que menos han sufrido las consecuencias de esta pandemia ya que, al contrario que otros bienes muebles expuestos en museos, no han necesitado de limpiezas o de desinfecciones con productos posiblemente abrasivos y nocivos. A su vez, el cierre derivado del confinamiento domiciliario en primavera de 2020, junto con el reducido flujo de visitantes posterior, han contribuido a frenar el desgaste que la propia visita del público provoca, lo que en parte ha beneficiado a la conservación y protección de numerosos bienes arqueológicos $y$ yacimientos.

A pesar de ello, la normativa recomienda no desinfectar los bienes más de lo estrictamente necesario, y siempre evitando materiales que puedan deteriorar de cualquier forma el patrimonio.

\section{METODOLOGÍA Y MUESTRA: LOS YACIMIENTOS SELECCIONADOS}

El objetivo principal de este trabajo era comprobar los cambios del flujo del número de visitantes en yacimientos arqueológicos musealizados en el territorio gallego, y comparar los datos de visitas en el periodo estival del contexto prepandémico (2019), y del pandémico (2020), entre yacimientos al aire libre y lugares cerrados, como museos o centros de interpretación, para así averiguar si la tendencia en este nuevo paradigma fue a favor de los centros al aire libre, ya que por sus características intrínsecas garantizan un porcentaje mayor de seguridad ante la posibilidad de contagio. Por tanto, el proceso ha seguido una metodología mixta, ya que no solamente se extrajeron datos cuantificables de los centros, sino que éstos fueron procesados 
de manera que tuvieron en cuenta otros factores que pudieran influir en los resultados.

La primera fase consistió en establecer unos criterios de selección de acuerdo con las características de los yacimientos y museos: en primer lugar, debían ser centros en Galicia con arte rupestre al aire libre, principalmente grabados rupestres de la prehistoria reciente en Galicia, que abarca desde el megalitismo hasta la Edad del Hierro (4000 a. de C. y I d. de C., aproximadamente), a los que también se han incluido fortificaciones in situ de la misma época como castros, ya que suponen los elementos principales del modelo de gestión patrimonial y turística que se quiere resaltar por sus características. Por tanto, y para que la comparativa fuera lo más completa posible, se escogieron sitios al aire libre y cerrados, con el objetivo de determinar si esto fuera un factor clave a la hora de decisión de los visitantes.

Consecuentemente, la lista se compone de sitios al aire libre con un centro de interpretación en el cual se puede contabilizar el número total de visitantes al área arqueológica, y museos cerrados en los cuales se exponen objetos, monumentos y artefactos prehistóricos dentro del mismo contexto histórico de la prehistoria reciente gallega.

Se descartaron aquellos lugares que no cuentan con ningún método de recuento de visitas, y también aquellos yacimientos o estaciones sin una estructura de acogida a visitantes o con un nivel básico de musealización; así como aquellos que por cuestiones de incompatibilidades de tiempo por el procesado de datos no han podido ser parte de la lista final. A su vez, no se contaron otros lugares que, a pesar de ser puntos de referencia dentro del ámbito del arqueoturismo gallego, no corresponden al periodo histórico que se estableció.

La segunda fase, es decir, la recopilación de datos, a pesar de ser una muestra pequeña, supuso un trabajo de meses ya que su obtención dependía de muchos factores e incidencias habituales en este tipo de procesos. El método principal fue la solicitud de datos de visita a los diferentes lugares correspondientes con las variables antes mencionadas.

Por último, en una tercera fase, se realizó un estudio de estos, en el cual los principales instrumentos de trabajo y volcado de información fueron tablas en Excel que, tras un proceso de análisis, resultaron gráficas y tablas (véase apartado de análisis y comparativa de resultados de este artículo), en las cuales se indicaban las tendencias de cada año y cada mes y se traducían de manera gráfica en estos elementos para pasar a su posterior interpretación.

Como ya se ha indicado, hay que tener en cuenta que la gran mayoría de estos lugares en Galicia no cuenta con medios para controlar los aforos o el 


\section{SITIOS AL AIRE LIBRE}

Parque Arqueológico de Campo Lameiro o PAAR (Praderrei, Pontevedra)

Área Arqueológica de Mogor (Marín, Pontevedra)

Parque Arqueológico de la Cultura Castreña de Ourense o PACC (Puxín, Ourense)

Castro de Viladonga (Castro de Rei, Lugo)

Castro de Elviña (A Coruña, A Coruña)

Castro de Santa Tegra (Oia, Pontevedra)

\section{SITIOS CERRADOS}

Museo Provincial de Lugo (Lugo, Lugo)

Centro Arqueológico del Dolmen de Dombate (Bergantiños, A Coruña)

Museo Arqueológico de Ourense- Sala de Exposiciones de San Francisco "Escolma de Escultura" (Ourense, Ourense)

Museo de Pontevedra- Edificios Sexto y Sarmiento (Pontevedra, Pontevedra)

Museo Arqueológico del Castillo de San Antón (A Coruña. A Coruña)

Centro de Interpretación de A Caeira (Poio, Pontevedra)

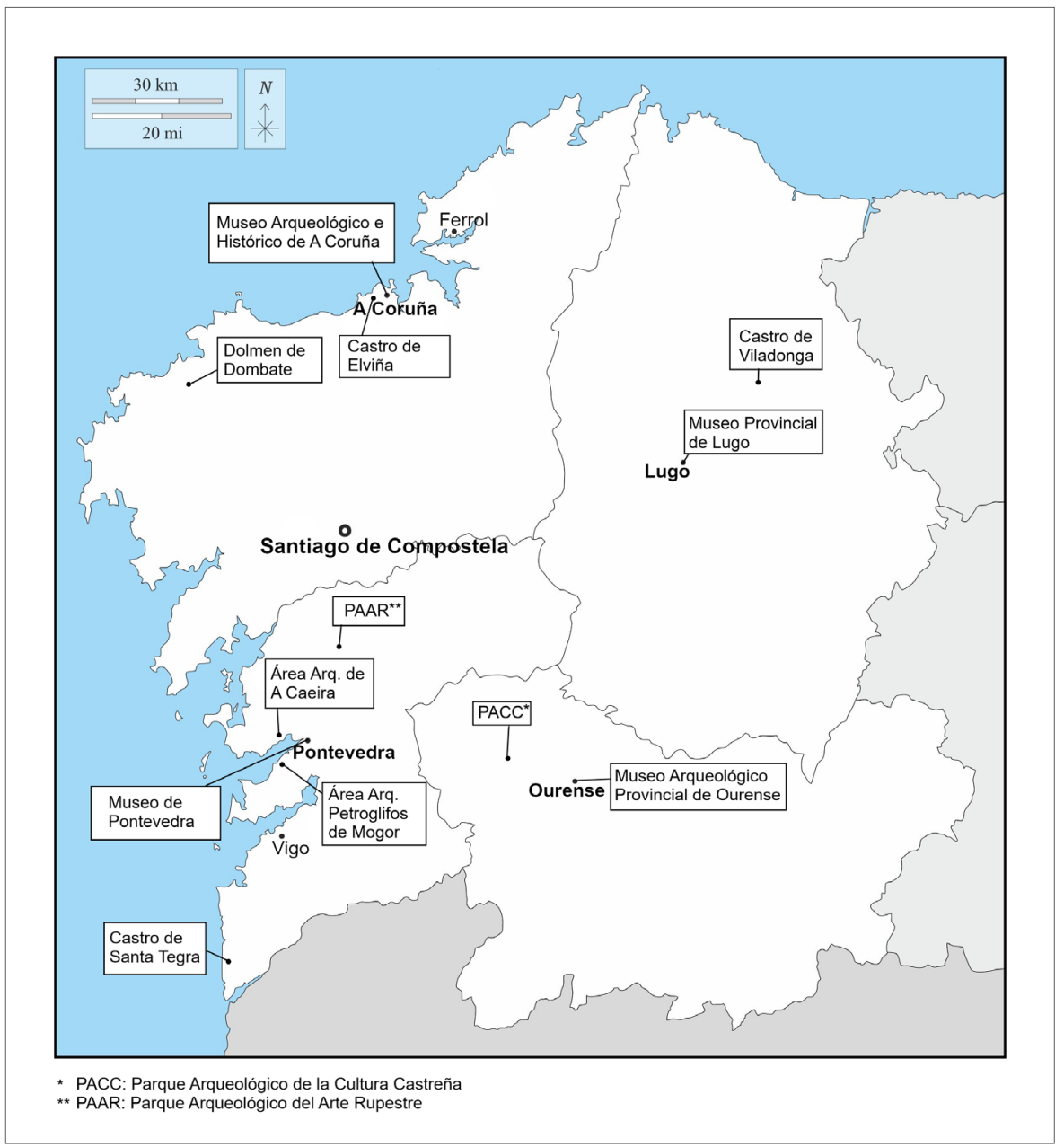


número de personas que acuden a visitar un monumento de estas características, precisamente por su ubicación al aire libre, y normalmente en fincas privadas o en montes comunales a los que cualquier persona puede acceder. Suelen ser fortificaciones o estaciones que solamente se han adaptado a las visitas con un área de acceso y/o señalizaciones y paneles informativos, o bien sin ninguno de estos elementos.

Al mismo tiempo, existen muchos otros yacimientos al aire libre con centros o áreas de interpretación en el entorno cercano, pero no adyacente a estos, por lo que resulta imposible contabilizar las visitas que reciben ya que una gran parte del público decide visitar el área sin pasar por el centro de interpretación, por tanto, no se han podido tener en cuenta para este artículo.

Esto incluye algunos de los casos estudiados, como es el caso del Área Arqueológica de A Caeira y su centro de interpretación que, debido a la separación del centro con el área, y el libre acceso de esta última, no se ha podido llevar a cabo un recuento de la misma, sino solamente del centro, y es por eso que se ha incluido como centro cerrado y no al aire libre a pesar de que cumple con ambos requisitos.

Esta circunstancia se repite incluso en áreas en las cuales el centro se encuentra dentro del mismo recinto de visita, como ocurre en el Área Arqueológica de los Laberintos de Mogor, en la cual no se ha podido realizar un recuento exacto de los visitantes ya que hay casos en los que se visitan los grabados, pero no el centro.

Por otro lado, en algunos sitios cerrados solamente se han incluido los datos de las salas o áreas de los centros dedicadas a la prehistoria y su arte, como es el caso del Museo Arqueológico de Ourense y el Museo de Pontevedra.

En definitiva, cada centro es diferente en cuanto a tamaño, capacidad de acogida y recepción de visitantes que, por supuesto se ve mermada por las medidas de distanciamiento social, y por otras razones como las que se han mencionado que al final afectan al número final de visitantes, y estas son incidencias que se han de tener en cuenta a la hora de interpretar los datos.

Los lugares seleccionados para este artículo corresponden a los requisitos indicados y son referencia en materia de turismo cultural arqueológico y prehistórico en Galicia. Es importante también mencionar que algunos centros de estas características no se han incluido por no poder acceder a los datos de visitantes, por motivos de infraestructura, o bien por incompatibilidades por razones de fechas de procesado de datos. 


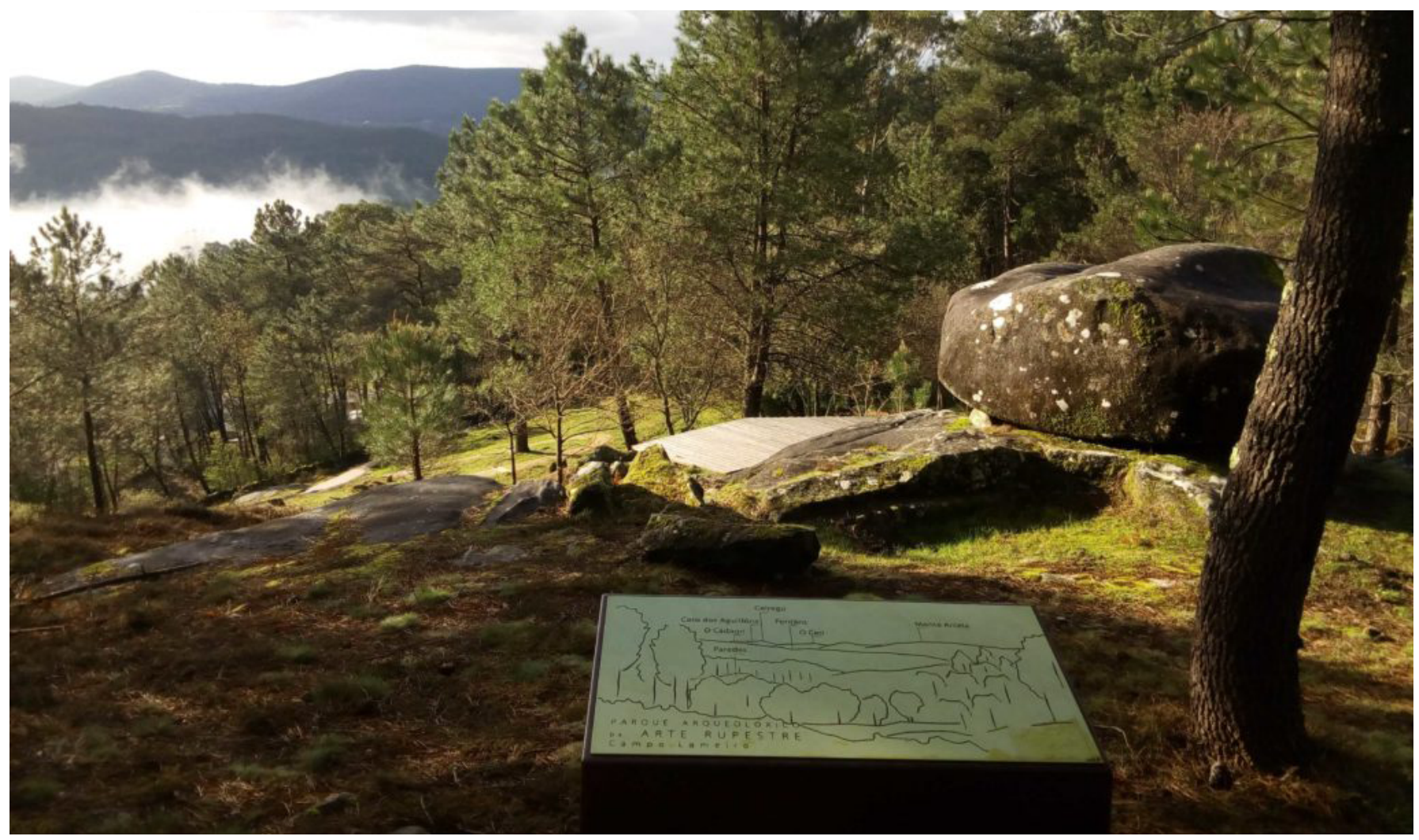

Vista panorámica del área al aire libre del Parque Arqueolóxico da Arte Rupestre | foto PAAR (Oldamkmj, 2019)

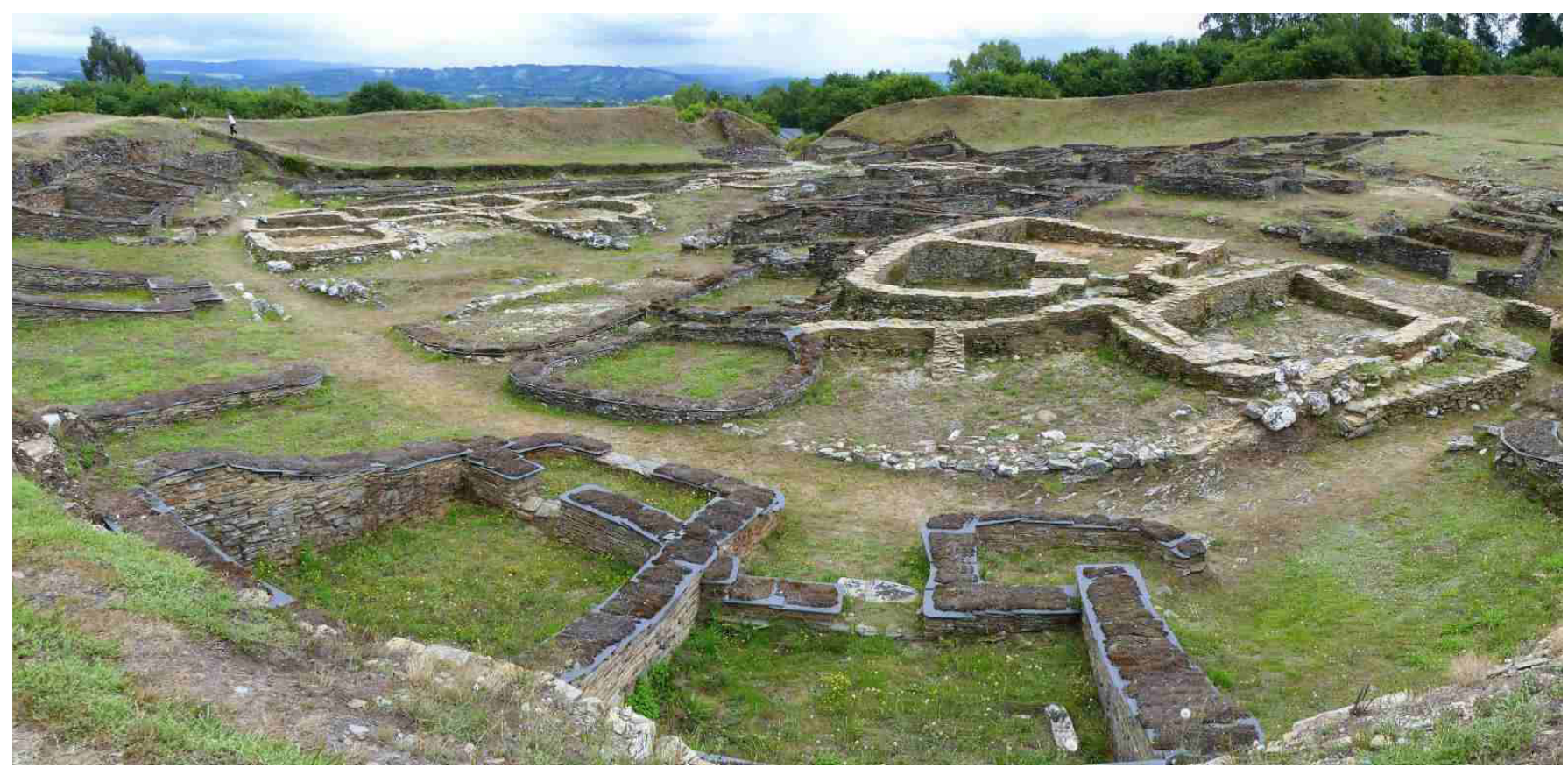

Vista del interior del castro de Viladonga desde la entrada principal | foto ArqueoTrip Guía Online de Turismo Arqueológico y Cultural, 2018 
Visitantes observando la exposición de arqueología y Prehistoria en el Museo de Pontevedra | foto La Voz de Galicia (Claudia Gómez)
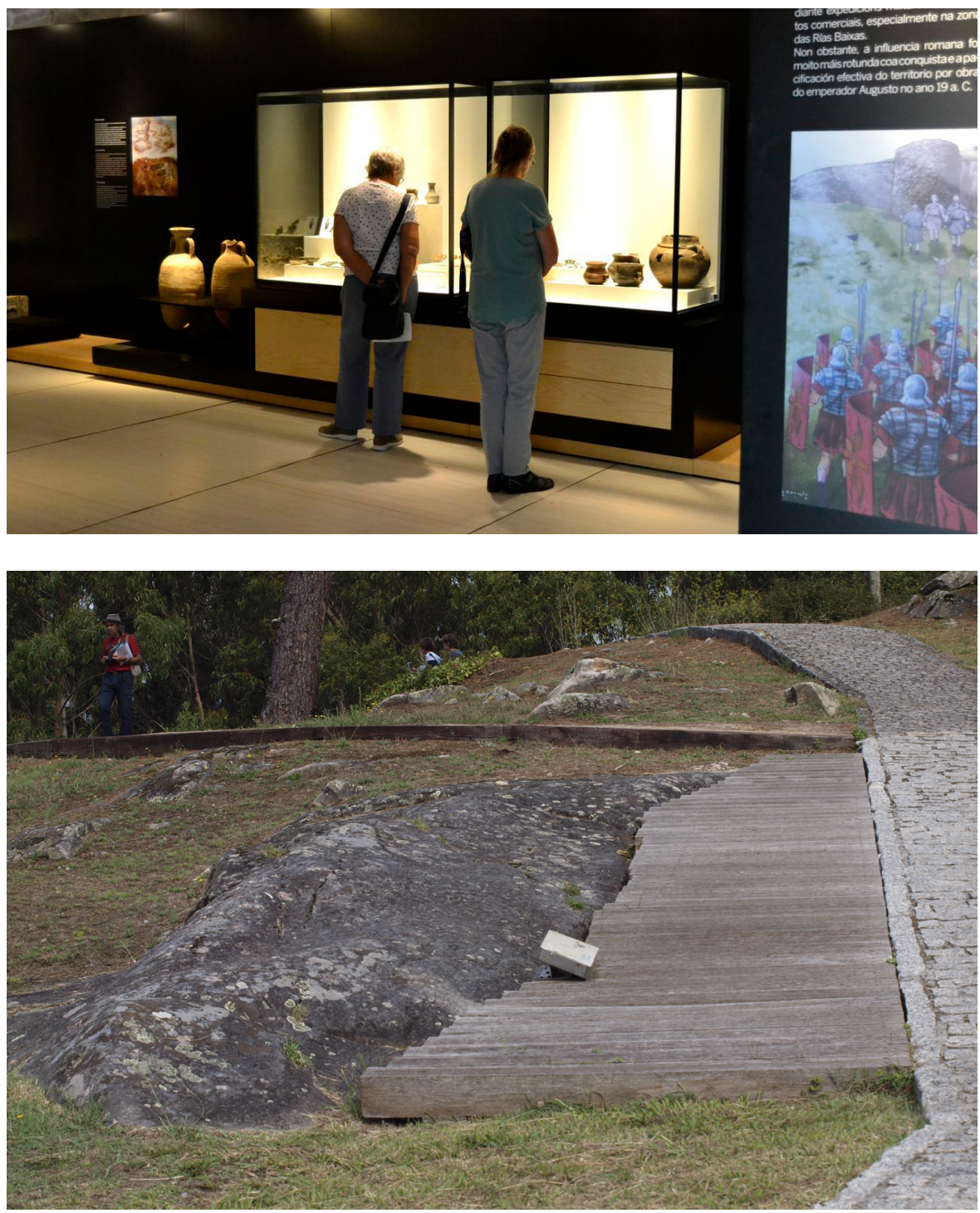

Recorrido con pasarelas de madera en el área arqueológica de Mogor (Pontevedra) | foto Estrela c. García García

Los yacimientos o lugares al aire libre son espacios diáfanos, marcados con paneles de madera, señalizaciones y una adaptación adecuada del terreno para permitir el paso cómodo del visitante mientras que se mantiene dentro de lo posible el impacto paisajístico de las estructuras de acomodación de los yacimientos.

Tal y como se puede comprobar en los yacimientos al aire libre, se trata de espacios abiertos, en los cuales existe un área, de mayor o menor tamaño dependiendo de la extensión del yacimiento, en la cual a la entrada se encuentra un centro de interpretación musealizado donde se establece un contexto historiográfico y arqueológico del lugar con un recorrido interior que 

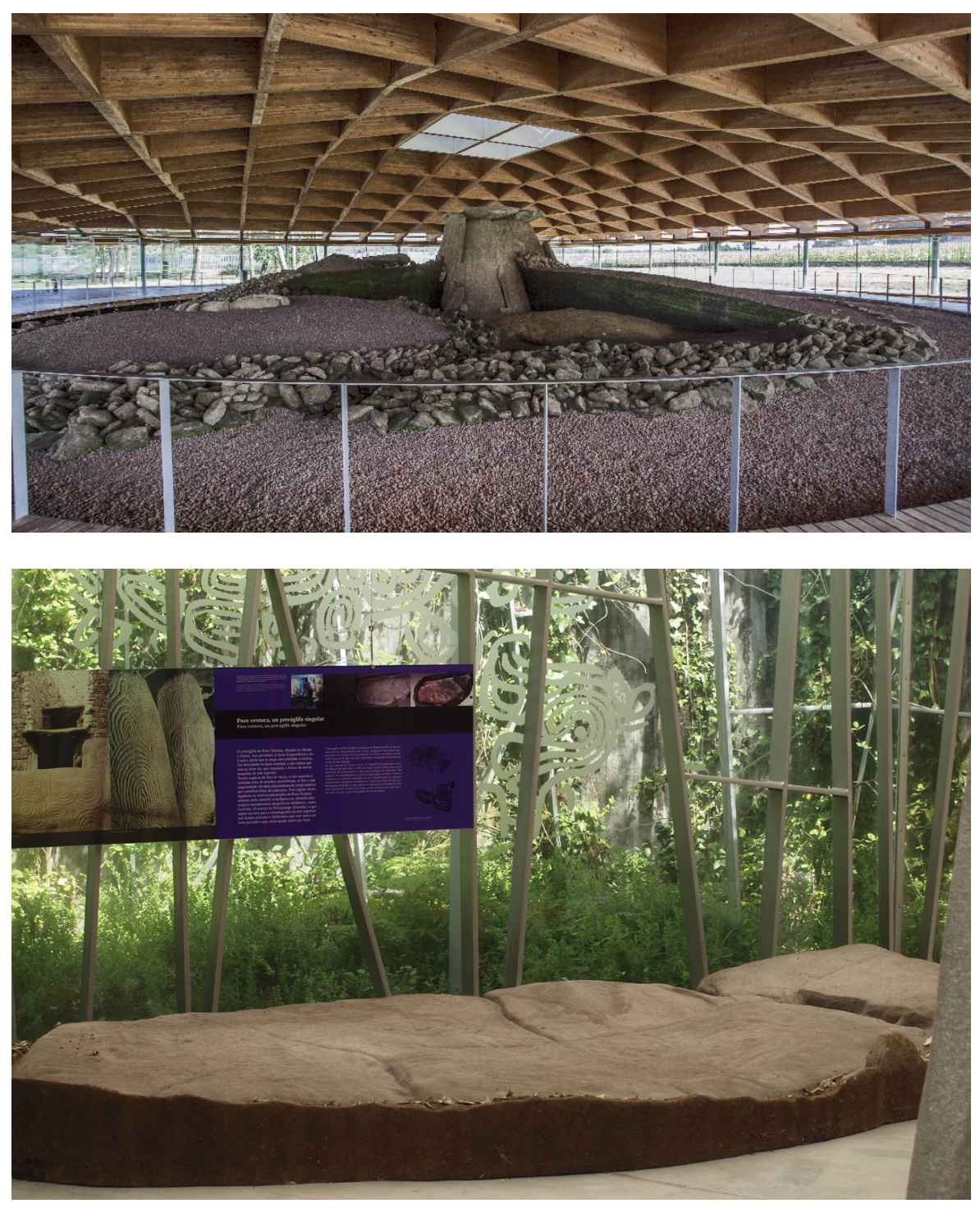

puede ser libre o guiado, en el que se incluyen otras acomodaciones propias de este tipo de centros como un área de recepción, servicios de recreo, etc.

Tras esta primera recepción le sigue un recorrido al aire libre, lugar en el cual se pueden visitar los bienes in situ dentro de su contexto original, y que está adaptado gracias a elementos como paneles, pasarelas y cartelería, que a su vez se puede recorrer por libre o en pequeños grupos con guía.

Este modelo de visita es muy fácilmente adaptable a las recomendaciones sanitarias que se han establecido desde el Ministerio de Cultura y otras instituciones, puesto que requiere de muy pocos cambios para poder disfrutar
Interior del Centro de Interpretación del Dolmen de Dombate en Bergantiños, A Coruña | foto Turismo de Galicia
Réplica del petroglifo de Pozo Ventura dentro del centro de interpretación de A Caeira (Pontevedra) | foto Estrela C. García García 
de una experiencia segura y satisfactoria, ya que la distancia interpersonal, la ventilación y la distancia con los propios bienes está garantizada de por sí, cosa que no sucede en un museo, o incluso en un entorno cerrado como una cavidad.

Sin embargo, en estos lugares cerrados, que corresponden con el modelo de visita tradicional a un espacio musealizado, se deben tomar muchas otras medidas que ya hemos visto, para poder adaptar la experiencia del visitante a las recomendaciones sanitarias, lo que puede suponer un factor en contra a la hora de recibir visitas.

Por supuesto, y como se ha comprobado, estos elementos no tienen por qué ser un hándicap, ya que si se siguen las recomendaciones adecuadamente el entorno no tiene por qué presentar ningún riesgo tanto para visitantes como para la plantilla.

\section{ANÁLISIS Y COMPARATIVA DE RESULTADOS}

Tras esta aportación contextual, pasaremos a analizar los datos de visitantes de los centros listados, y ver cuáles han sido los números, a través de los gráficos de las páginas siguientes.

Se puede comprobar un descenso en el número de visitantes tanto en lugares al aire libre como cerrados, sin embargo, el descenso es mucho más acusado en estos últimos. En áreas con yacimientos prehistóricos al aire libre el mayor descenso de visitantes entre los meses de verano de 2019 y 2020 es del $55 \%$ en el caso del Castro de Elviña, lo que da un descenso de media del $36 \%$; mientras que en lugares cerrados se llegan a cifras tan elevadas como el $92 \%$ de reducción del Museo Provincial de Lugo, aumentando la media de descenso de visitantes hasta el $54 \%$. Se aprecia una diferencia clara entre lugares al aire libre y lugares cerrados.

Por tanto, a simple vista, el descenso ha sido generalizado, a excepción de casos muy concretos como es el del Centro de Interpretación del Dolmen de Dombate en el cual la reducción de las visitas fue de un 2 \%. Es importante subrayar dos factores significativos: su condición de estar localizado in situ, y la propia concepción del centro, que hace una percepción de espacio abierto, que probablemente han influenciado en la mínima reducción del número de visitantes en contraste con los otros centros.

El descenso de estas cifras está provocado indudablemente por efectos de la pandemia y las medidas restrictivas que afectaron a la movilidad y el turismo durante el periodo estival, que en el caso gallego fueron distintas según la incidencia de casos en diferentes áreas sanitarias. A pesar de ello, 

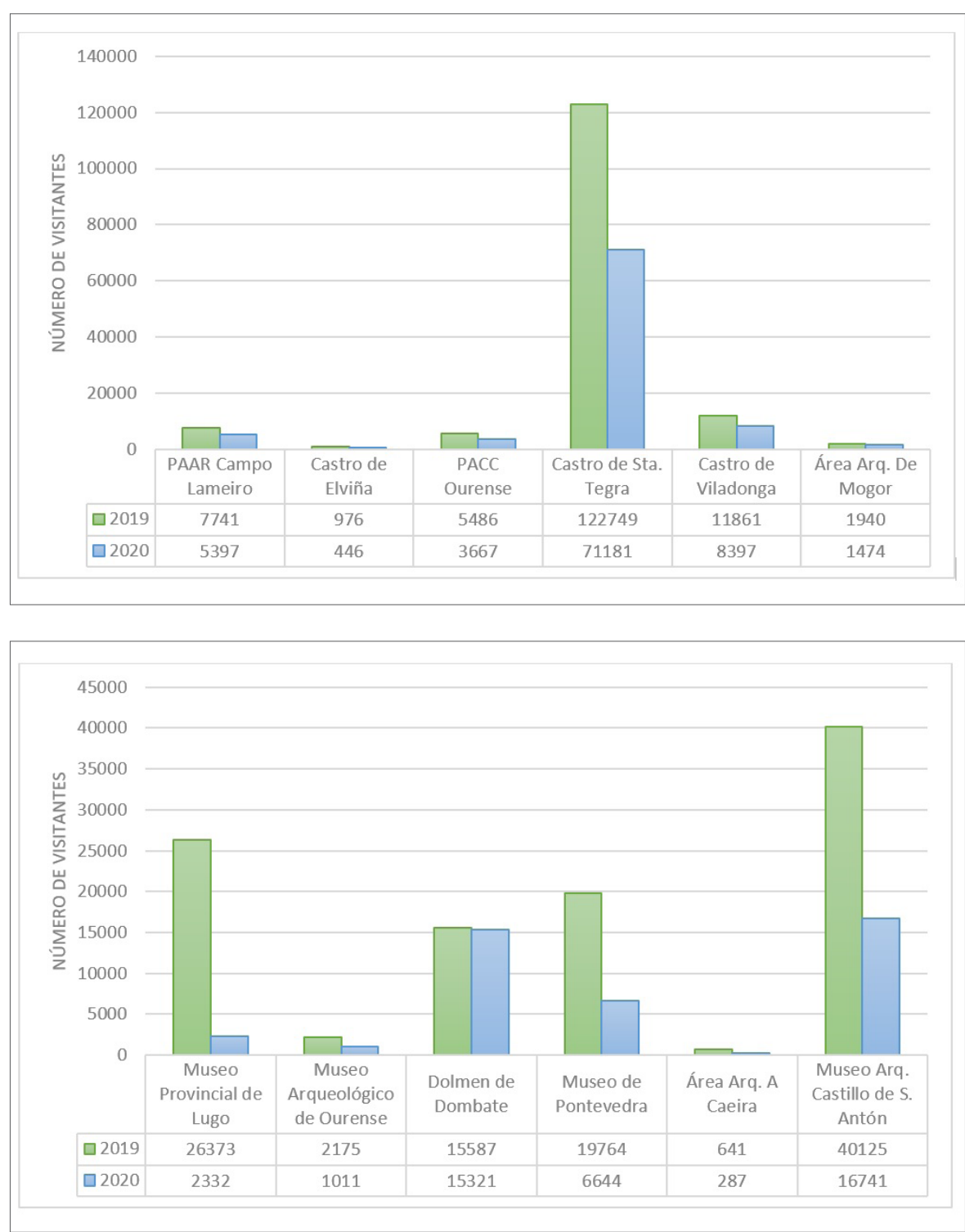

durante el periodo estival hubo un menor número de restricciones, y salvo algunas excepciones, la movilidad fue libre en todo el territorio gallego, con el objetivo de recuperar las pérdidas económicas del segundo trimestre y evitar una fuerte caída en la campaña de verano.

Hubo mayores restricciones en los centros culturales, que redujeron aforo, y sufrieron una reestructuración del plan de visitas y actividades para acomodarlo a las recomendaciones que hemos visto antes. Esto supuso en parte un detrimento en el número de visitantes, a lo que se suma la situación sanitaria.
Comparativa de número de visitantes durante los periodos estivales de 2019 y 2020 en lugares con arte y/o yacimientos prehistóricos al aire libre en Galicia | gráfico Estrela C. García García
Comparativa de número de visitantes durante los periodos estivales de 2019 y 2020 en museos y centros culturales cerrados con arte y/o bienes prehistóricos | gráfico Estrela C. García García 
Comparativa de número de visitantes durante los periodos estivales de 2019 y 2020 en lugares con arte y/o yacimientos prehistóricos al aire libre en Galicia | gráfico Estrela C. García García
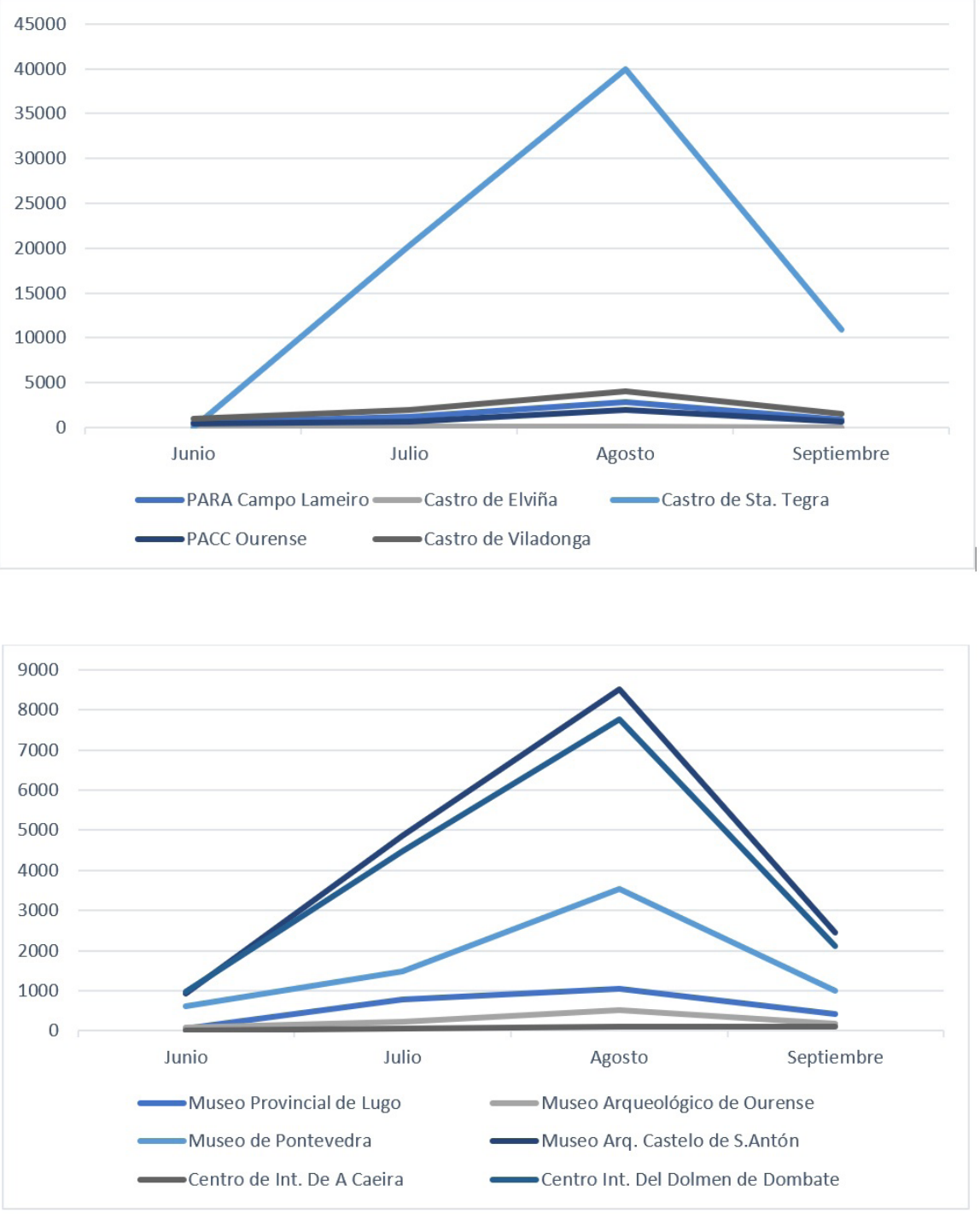

Comparativa de número de visitantes durante los periodos estivales de 2019 y 2020 en museos y centros culturales cerrados con arte y/o bienes prehistóricos | gráfico Estrela C. García García
Hay otros factores a tener en cuenta como es la meteorología, que en verano de 2020 fue excepcionalmente buena en Galicia con presencia de aire cálido venido de África y tendencia anticiclónica (Xunta de Galicia, Meteogalicia $2020,4-5)$, favoreciendo el arqueoturismo y visitas a yacimientos al aire libre, en detrimento de museos o centros cerrados.

Comprobando también el flujo de visitantes en los mismos centros, se puede apreciar que la tendencia es ascendente entre los meses de junio y agosto, llegando entonces el pico de visitas, y seguidamente experimentando una reducción en algunos casos drástica en septiembre, cuando la gran mayoría de la población se reincorpora al trabajo. Comparando los datos en este 
caso es evidente que las tendencias son las mismas y los visitantes se comportan de un mismo modo a pesar de visitar lugares con distintas características y modelos de turismo cultural.

Es interesante también destacar que los flujos de visita no coinciden exactamente con el aumento de los casos, que en el mismo periodo estival sufrió un ascenso mensual generalizado, llegando al máximo a principios de septiembre (Mapa 2020). Es decir, que el aumento de los casos, a pesar de tener influencia a la hora de este descenso, no fue un elemento determinante, ya que no se produce una bajada del número de visitantes a medida que los casos activos aumentaban.

Volviendo a los primeros dos gráficos, los datos demuestran que el visitante se ve atraído por lugares al aire libre. A pesar de que los números tienden a la baja -quizás por mayor desconocimiento generalizado de estos enclaves, no olvidemos que el turismo arqueológico no es el principal tipo de turismo en el periodo estival-, se puede apreciar que en lugares arqueológicos al aire libre el descenso es significativamente menor ya que las características intrínsecas que lo conforman lo hacen más atractivo y se percibe de forma distinta, más segura.

El aire libre proporciona, como es lógico, la ventilación natural del lugar, reduciendo el riesgo de contagio por aerosoles que en una sala cerrada sería mayor. Por otra parte, mantener las distancias interpersonales es tarea mucho más fácil, incluso con visitas guiadas o en grupo.

Es también beneficioso para el personal trabajador y de limpieza que, asegurando la distancia del visitante con los bienes, puede garantizar que estos no necesitarán de limpieza desinfectante especial, que podría ser potencialmente dañina para la superficie e integridad de este tipo de patrimonio arqueológico, que por sí mismo es extremadamente frágil por su antigüedad.

Esta situación excepcional también ha sacado a relucir una realidad que desde hace años se viene debatiendo en el ámbito del turismo cultural y arqueológico, que es el turismo de masas. Gracias a la reducción de aforos, y por supuesto el cierre total del confinamiento domiciliario de marzo de 2020, el desgaste y la erosión naturales se han visto reducidas debido al menor número de afluencia en estos lugares. La presencia de grandes masas de gente es un agente nocivo para el arte rupestre y los yacimientos arqueológicos de estas características, que puede ser un factor clave en su deterioro rápido y su posible destrucción. Son las propias características del patrimonio arqueológico, en especial del prehistórico, las que obligan a una reducción de los aforos para asegurar su conservación a largo plazo, y es precisamente esto lo que ha atraído al turismo a estos lugares. 
La presencia de un menor número de visitantes ha sido un soplo de aire fresco en estos enclaves, y ha demostrado que otro modelo de turismo arqueológico es posible, uno en el cual se mantiene mayor equilibrio entre bienestar del yacimiento y del público.

Muchas organizaciones y centros culturales han repensado y se han replanteado el modelo de negocio que hasta ahora llevaban a cabo a raíz de la pandemia (Abeledo Sanchís 2020, 26), lo que demuestra que la idea de otro modelo de visita más sostenible y respetuoso con el patrimonio ha venido para quedarse.

No es posible decir qué ocurrirá a nivel global con el modelo de turismo masivo que se ha promocionado en las últimas décadas, pero estos datos muestran una tendencia que apunta a un cambio. La pandemia de coronavirus ha sido un evento a escala global que ha obligado a reducir la afluencia de gente que visita un sitio o monumento y que ha potenciado a su vez el turismo local.

Es en estos lugares al aire libre donde mejor se ha reflejado este concepto, y donde se ha podido comprobar que son un atractivo mayor para un perfil de visitante que busca un tipo de turismo más específico y seguro que acudir a playas u otras zonas de ocio con mayor concentración de personas.

Por tanto, estos enclaves el son ejemplo perfecto para llevar a cabo este tipo de modelo de arqueoturismo más equilibrado, y tal y como se ha podido comprobar, ha resultado útil en un periodo estival como nunca antes se había visto. A pesar de la reducción de visitas, los centros culturales se han conseguido mantener, y han demostrado que es posible hacer sobrevivir la cultura a la vez que se protege la salud pública, haciendo que las perspectivas de futuro para los próximos veranos sean más prometedoras.

\section{CONSIDERACIONES FINALES}

Tras examinar estos datos, se hace patente la necesidad de apostar por este modelo de visitas más seguro y sostenible, que no solamente mantiene viva la cultura, sino que puede revitalizar la difusión del patrimonio arqueológico prehistórico al aire libre, en detrimento del modelo de turismo de masas. Se ha comprobado que sus características proporcionan un entorno más seguro y/o atractivo al visitante, y que es vital, en un contexto de tan grande incertidumbre ocasionada por la situación de crisis sanitaria y social, encontrar formas de mantener vivo el patrimonio garantizando la seguridad de todas las partes implicadas.

Realmente podría suponer un antes y un después, y una solución para asegurar la conservación y promoción del patrimonio arqueológico, que siempre 
resulta un agente dañado en cualquier situación de crisis. Ahora se presenta una oportunidad única de crear una nueva forma de asumir el turismo y desarrollar un modelo más adecuado al contexto que se presenta a corto, medio y largo plazo.

Los datos que se han analizado demuestran que, a pesar de las circunstancias adversas, el público sigue interesado en visitar estos lugares. Ya se ha hecho hincapié en el hecho de que estos datos son aproximados, puesto que la mayor parte de estaciones con arte prehistórico al aire libre o yacimientos prehistóricos en Galicia se encuentran en áreas apartadas en ámbito rural y sin sistemas de contabilización que nos puedan indicar cuál ha sido el aforo de visitantes durante el pasado verano de 2020 ni ningún otro.

A pesar de ello, es vital tener en cuenta estos datos y analizarlos más a fondo, con el objetivo de desarrollar un plan para el verano del año 2021 y los futuros periodos estivales, de manera que se pueda asegurar la sostenibilidad y longevidad de este modelo de cara al futuro.

A esto se añade una circunstancia especial que se ha observado durante el periodo estival del 2020: el crecimiento de la afluencia de personas en entornos rurales. El público busca alejarse de la multitud y de las grandes aglomeraciones de gente, en busca de espacios abiertos y diáfanos en los cuales el riesgo de contagio es menor. Según los datos de este pasado periodo estival el alquiler de alojamientos rurales fue mayor en un $6 \%$ que el alquiler de apartamentos turísticos o de playa (Basanta, 2020).

Esto ha hecho más atractivas otras formas de realizar turismo cultural, poniendo el turismo arqueológico al aire libre como uno de los grandes atractivos en Galicia. Por tanto, es fundamental invertir y promocionar nuestro patrimonio arqueológico a través de este modelo de visitas más seguro y que proporciona grandes perspectivas para el futuro de la puesta en valor del arte y de los yacimientos prehistóricos, que actualmente necesitan de especial atención y cuidado por su enorme fragilidad y abandono.

La promoción de un turismo arqueológico seguro, sostenible y más consciente con otros aspectos que preocupan en el presente como es la accesibilidad, la inclusión y el cambio climático puede ser la clave de asegurar la preservación de nuestro arte prehistórico y de promocionar nuestra cultura y nuestra historia a generaciones futuras.

\section{Agradecimientos}

Me gustaría agradecer su colaboración a todos los centros que han participado y han cedido estos datos, ya que sin su aportación no hubiera sido posible llevar a cabo este trabajo. 


\section{BIBLIOGRAFÍA}

- Abeledo Sanchís, R. (dir.) (2020) Análisis del impacto del COVID-19 sobre las organizaciones y agentes culturales en España. Valencia: Econcult, Universidad de Valencia. Disponible en: http://www.econcult.eu/wp-content/uploads/ 2020/07/Esp-covid-19 compressed.pdf [Consulta: 02/01/ 2020]

- Basanta, E. (2020) El verano de 2020 revoluciona el alquiler vacacional: más días reservados, más caro y más turismo rural. Idealista, 14 de julio de 2020. Disponible en: https://www.idealista.com/news/vacacional/mercado-vaca cional/2020/07/14/785106-el-verano-de-2020-revolucionael-alquiler-vacacional-mas-dias-reservados-mas [Consulta: 08/01/2021]

- Blázquez, R. (2020) ¿Es peligrosa la cultura en tiempos de covid? Sólo tres de los 11.600 brotes tienen vínculos con una actividad cultural. La Sexta Clave, 30 de octubre de 2020. Disponible en: https://www.lasexta.com/programas/ lasexta-clave/es-peligrosa-la-cultura-en-tiempos-de-covidsolo-tres-de-los-11600-brotes-tiene-vinculos-con-unaactividad-cultural_202010305f9c76b09a1c4e000160d453. html [Consulta: 12/12/2020]

- Fernández Ortea, J. (2017) Análisis cuantitativo del impacto del arqueoturismo en España. Revista Investigaciones Turísticas, n. ${ }^{\circ} 14$, pp. 87-108. Disponible en: https://rua.ua.es/dspace/bitstream/10045/72119/1/ Investigaciones-Turisticas_14_05.pdf [Consulta: 10 /04/2021]

- López Ortega, D. y Collado Moreno, Y. (2018) Arqueoturismo ¿Un fenómeno en auge? Reflexiones acerca del turismo arqueológico en la actualidad en España. Pasos. Revista de turismo y patrimonio cultural, vol. $16, \mathrm{n}{ }^{\circ}$ 3, pp. 599-615. Disponible en: http://www.pasosonline.org/ Publicados/16318/PS318_04.pdf [Consulta: 09/04/2021]

- El mapa y la evolución del coronavirus en Galicia (2020) Faro de Vigo. A Fondo. Disponible en: https://afondo. farodevigo.es/galicia/el-mapa-del-coronavirus-en-galicia. html [Consulta: 15/01/2021]

- Ministerio de Cultura y Deporte, Instituto de Patrimonio Cultural de España (2020) Pautas para la gestión y el disfrute público del patrimonio cultural en la desescalada de la crisis sanitaria (COVID-19). Madrid, mayo de 2020 Disponible en: https://ipce.culturaydeporte.gob.es/dam/ jcr:39bc357b-dcf2-4bd4-9ce6-eda384872c99/documentogesti-n-patrimonio-cultural-covid-19.pdf [Consulta: 08/12/ 2020]

- Ministerio de Sanidad (2020) Actualización n. 235. Enfermedad por el coronavirus (COVID-19). 23 de octubre de 20. Disponible en: https://www.mscbs.gob.es/ profesionales/saludPublica/ccayes/alertasActual/nCov/ documentos/Actualizacion_235_COVID-19.pdf [Consulta: $12 / 12 / 2020]$
- Paquete de ayudas económicas del Gobierno al sector cultural por la crisis del coronavirus (2020). El Mundo, 31 de marzo de 2020. Disponible en: https:// www.elmundo.es/cultura/2020/03/31/5e8367f22 1efa02f278b4663.html [Consulta 19/01/2021]

- Sandri, P.M. (2020) El sector de la cultura mundial arrastra números rojos que tardará años en superar. La Vanguardia, 2 de agosto de 2020. Disponible en: https://www.lavan guardia.com/cultura/20200802/482625416646/crisis-cultu ra-pandemia-coronavirus.html [Consulta:15/12/2020]

- Unesco (2020a) Cultura \& COVID-19. Impacto y respuesta, n. ${ }^{0} 4,13$ de mayo de 2020. Disponible en: https://es.unesco.org/sites/default/files/is sue_4_es_culture_covid-19_tracker-2.pdf [Consulta: 21 $112 \overline{2} 0 \overline{2} 0]$

- Unesco (2020b) Boletín semanal. Cultura y COVID-19. Impacto y respuesta, $n .^{\circ} 5,27$ de mayo de 2020. Disponible en: https://es.unesco.org/sites/default/files/isssue_5_es_ culture_covid-19_tracker-2.pdf [Consulta: 26 /12/2020]

- Unesco (2020c) Cultura y COVID-19. Impacto y respuesta. Número especial, n.o 7, 3 de julio de 2020. Disponible en: https://es.unesco.org/sites/default/files/ issue_7_es_culture_covid-19_tracker.pdf [Consulta: 26/12/2020]

- Xunta de Galicia, Meteogalicia (2020) Informe climatolóxico xullo de 2020. 8 de agosto de 2020. Disponible en: https://www.meteogalicia.gal/datosred/ infoweb/clima/informes/estacions/mensuais/2020/202007 es.pdf [Consulta: 05/12/2020] 